\title{
Estimativas de parâmetros genéticos para características de crescimento e produtividade em vacas da raça Canchim, utilizando-se inferência bayesiana ${ }^{1}$
}

\author{
Silvio de Paula Mello ${ }^{2}$, Maurício Mello de Alencar ${ }^{3}$, Fábio Luiz Buranelo Toral ${ }^{4}$, Viviane \\ Karina Gianlorenço ${ }^{5}$
}

\author{
${ }^{1}$ Trabalho realizado com o apoio financeiro do CNPq. \\ ${ }^{2}$ UFSCar, Departamento de Genética e Evolução. \\ ${ }^{3}$ Embrapa Pecuária Sudeste, Caixa Postal 339, São Carlos, SP, CEP: 13560-970. \\ ${ }^{4}$ UNESP - Jaboticabal, Departamento de Melhoramento Animal. \\ ${ }^{5}$ Universidade de Marília - Unimar, Departamento de Ciências Agrárias.
}

RESUMO - O objetivo neste trabalho foi estimar as herdabilidades e as correlações genéticas dos pesos à desmama (PD), aos 12 meses de idade (P12) e à idade adulta (PAD), da idade de descarte (TPR - tempo de permanência no rebanho), do número (ND10) e quilogramas (QD10) de bezerros desmamados em até dez anos de idade, do número total (NDT) e quilogramas totais (QDT) de bezerros desmamados durante todo o tempo de permanência no rebanho e dos quilogramas de bezerros desmamados por ano de permanência no rebanho (QTPR) de fêmeas de um rebanho da raça Canchim. As estimativas dos componentes de (co)variância foram obtidas pelo método bayesiano, em análises bicaráter de PD, P12 e PAD com as demais características. Os modelos estatísticos incluíram os efeitos aleatórios genético aditivo direto, de ambiente permanente e residual e os efeitos fixos de ano e mês de nascimento ou do parto, da idade da vaca ao parto e da idade do animal, dependendo da característica. As médias das estimativas de herdabilidade foram iguais a 0,38 (PD), 0,40 (P12), 0,54 (PAD), 0,22 (TPR), 0,22 (ND10), 0,24 (QD10), 0,23 (NDT), 0,23 (QDT) e 0,32 (QTPR), indicando que as características possuem variação genética aditiva suficiente para boa resposta à seleção. As correlações genéticas entre TPR $(-0,02 ; 0,26$ e $-0,12)$, ND10 (0,04; 0,10 e $-0,29)$, QD10 (0,37; 0,39 e -0,13), NDT (-0,03; 0,14 e -0,25), QDT $(0,20 ; 0,33$ e -0,16) e QTPR $(0,21 ; 0,28$ e $-0,19)$ e os pesos (PD, P12 e PAD), ao contrário do aumento do peso adulto, sugerem que a seleção de fêmeas com base nos pesos à desmama e aos 12 meses de idade não deve prejudicar as características de longevidade e de produtividade estudadas.

Palavras-chave: bovinos de corte, longevidade de fêmeas, peso, produtividade de fêmeas

\section{Estimates of genetic parameters for growth and productivity traits of Canchim cows using Bayesian inference}

\begin{abstract}
The objective of this study was to estimate genetic parameters for body weights at weaning (PD), 12 months old (P12) and adult age (PAD), culling age (TPR, days in herd), number (ND10) and kilograms (QD10) of calves weaned up to ten years of age, total number (NDT) and total kilograms (QDT) of calves weaned during herd life, and kilograms of calves weaned per year in herd (QTPR) of Canchim (5/8 Charolais + 3/8 Zebu) females from one herd. Data consisted of 3,249, 3,111, $1,138,1,340,1,362,1,362,1,340,1,340$ and 1,340 records of PD, P12, PAD, TPR, ND10, QD10, NDT, QDT and QTPR, respectively. Variance and covariance components were estimated by bivariate analyses between PD, P12 and PAD and other production traits using Bayesian inference. The models included the additive direct, permanent environmental and residual random effects and the fixed effects year and month of birth or calving, calving age and age of the animal, depending on the trait. QD10, QDT and QTPR of each female were obtained by adjusting the weaning weights of calves for year and month of birth, sex and age of cow. Average of heritability estimates were 0.38 (PD), 0.40 (P12), 0.54 (PAD), 0.22 (TPR), 0.22 (ND10), 0.24 (QD10), 0.23 (NDT), 0.23 (QDT) and 0.32 (QTPR), indicating genetic variability to obtain response by selection. Genetic correlations between TPR (-0.02, 0.26 and -0.12), ND10 (0.04, 0.10 and -0.29), QD10 (0.37, 0.39 and $-0.13)$, NDT (-0.03, 0.14 and -0.25$)$, QDT (0.20, 0.33 and -0.16$)$, QTPR (0.21, 0.28 and -0.19$)$ and body weights (PD, P12 and PAD) suggest that selection of females based on weaning and 12-month body weights will not affect productivity. However, it may be decreased by increasing female adult body weight.
\end{abstract}

Key Words: beef cattle, body weight, days in herd, female productivity

\section{Introdução}

A taxa de desfrute do rebanho bovino brasileiro é baixa (próxima de 20\%) em relação às de países desenvolvidos (30 a 35\%) (Anualpec, 2003). Entre os fatores inibidores da produção de carne bovina no Brasil, encontram-se aqueles inerentes à alimentação, à sanidade, ao manejo e ao potencial genético. 
Um dos recursos para se modificar o potencial genético dos rebanhos é a seleção, que depende de programas de avaliação genética bem estruturados. Nas duas últimas décadas, vários programas de melhoramento foram implementados no Brasil para várias raças bovinas de corte, incluindo vários tipos de características nas avaliações genéticas. O peso, avaliado em diferentes idades, é uma característica de crescimento largamente utilizada como critério de seleção nesses programas (Alencar, 2002). Entretanto, há evidências de correlação genética positiva e alta entre pesos em idades jovens e os pesos à idade adulta e à maturidade de fêmeas (Silva et al., 2000; Talhari et al., 2003), sugerindo que a utilização de peso como critério de seleção pode resultar em aumento no tamanho das vacas e, segundo Lanna \& Packer (1998), o aumento do peso da vaca adulta além do suportável pelo programa nutricional ocasiona problemas reprodutivos e rápido declínio nos índices de produtividade da vaca. Alguns pesquisadores iniciaram, recentemente, o estudo genético de características de longevidade (Mercadante et al., 2000, Mello et al., 2002b; Gianlorenço et al., 2002) e produtividade (Barbosa et al., 2002; Alencar et al., 2003; Gianlorenço et al., 2003) no Brasil, que poderão futuramente ser incluídas em programas de avaliação genética.

O objetivo neste trabalho foi estimar as herdabilidades e as correlações genéticas de características de peso, longevidade e produtividade de fêmeas em um rebanho da raça Canchim, utilizando-se inferência bayesiana.

\section{Material e Métodos}

Os dados utilizados neste estudo são provenientes de animais do rebanho de bovinos da raça Canchim pertencente à Embrapa Pecuária Sudeste, localizada no município de São Carlos, região central do estado de São Paulo. Foram utilizados dados de fêmeas criadas exclusivamente em regime de pastagens, recebendo suplementação mineral durante todo o ano e os cuidados sanitários normais adotados na região. O rebanho estudado é fechado desde sua formação.

O manejo reprodutivo a que as fêmeas deste rebanho foram submetidas variou com o passar dos anos. Até 1975, as novilhas normalmente iniciaram a vida reprodutiva com aproximadamente 34 meses de idade e $360 \mathrm{~kg}$ de peso vivo (PV). Em 1976, este critério foi modificado para 24-28 meses de idade e $300 \mathrm{~kg}$ de PV. Até 1968 , as vacas eram colocadas com os touros após a desmama dos bezerros (sete a oito meses). Esse manejo foi modificado em 1969, quando todas as vacas que pariam antes do início da estação de monta foram colocadas em reprodução, e novamente em 1976, quando todas as vacas passaram a ser colocadas com touros durante a estação de monta. Durante as estações de monta, em torno de 30 fêmeas por touro eram colocadas em piquetes isolados e, a partir de 1979, deu-se início à utilização de inseminação artificial. Durante vários anos foram utilizadas duas estações de monta, uma no primeiro semestre e outra no segundo semestre, sem um mês fixo para iniciar ou terminar, e, portanto, com duração também variável (2 a 4 meses).

Os critérios de seleção e de descarte de animais do rebanho também variaram com o passar dos anos. A escolha das fêmeas para reprodução era realizada à época da desmama ( 8 ou 9 meses de idade), ao completarem um ano ou um ano e meio de idade, e aos dois ou dois anos e meio de idade. A partir de 1979, a seleção de novilhas para reprodução passou a ser feita antes do início da estação de monta, por volta dos 22 meses de idade, considerando-se, além de características raciais, o desenvolvimento ponderal dos animais. A eliminação de vacas do rebanho foi conseqüência principalmente de doenças e/ou acidentes. Contudo, a partir de 1977 iniciou-se o descarte de vacas consideradas de fertilidade mais baixa, ou seja, que saíssem vazias de duas estações de monta consecutivas. Procurou-se manter no rebanho apenas novilhas prenhes da primeira estação de monta.

As características estudadas nas fêmeas foram: peso à desmama (PD); peso aos 12 meses de idade (P12); peso à idade adulta (PAD); tempo de permanência no rebanho (TPR); número (ND10) e quilogramas (QD10) de bezerros desmamados em até 10 anos de permanência no rebanho; número (NDT) e quilogramas (QDT) de bezerros desmamados durante a permanência no rebanho; e quilogramas de bezerros desmamados por ano de permanência no rebanho (QTPR).

Os pesos à desmama e aos 12 meses de idade foram padronizados para 240 e 365 dias de idade, respectivamente, utilizando-se, na mesma ordem, 3.249 e 3.111 animais nascidos de 1958 a 2001.

O PAD correspondeu ao peso logo após o parto, para vacas de 4 a 10 anos, considerando-se apenas um peso para cada vaca e, no caso de a vaca possuir mais de um peso, considerou-se aquele mais próximo dos seis anos de idade. Foram utilizados dados de 1.138 vacas nascidas de 1967 a 1998 e paridas de 1977 a 2002.

A característica TPR correspondeu à idade, em dias, em que o animal foi descartado do rebanho, enquanto ND10 e NDT, ao número de bezerros efetivamente desmamados em até 10 anos de idade e até a saída da vaca do rebanho, respectivamente.

Para calcular o QD10 e o QDT de cada fêmea, primeiramente foi realizada análise de variância dos pesos à 
desmama de seus bezerros, padronizados para 240 dias de idade, pelo método dos quadrados mínimos, adotando-se modelo estatístico que incluiu os efeitos de ano e mês de nascimento do bezerro, sexo do bezerro e idade da vaca ao parto. Com base nessa análise, os pesos à desmama foram ajustados para todos esses efeitos antes de se calcularem o QD10 e o QDT de cada fêmea, que são a soma dos pesos à desmama ajustados de todos os bezerros da vaca, nascidos até que ela tenha completado dez anos de idade e durante todo o tempo que ela permaneceu no rebanho, respectivamente. A característica QTPR foi obtida dividindo-se QDT por TPR, em anos.

Para as características TPR, NDT, QDT e QTPR, foram utilizados os dados de 1.340 vacas nascidas de 1960 a 1990, uma vez que todos os animais nascidos até esse ano já deixaram o rebanho. Para ND10 e QD10 foram utilizados os dados de 1.362 vacas nascidas de 1958 a 1991, visto que os animais nascidos até esse ano já completaram dez anos de idade.

Para TPR, ND10, NDT, QD10, QDT e QTPR, foram consideradas apenas as observações de fêmeas que tiveram a oportunidade de conceber pelo menos uma vez e cuja saída do rebanho não foi por motivos de acidentes e/ou doença, mas por venda para abate ou reprodução ou por transferência.

Os componentes de (co)variância foram estimados pelo método bayesiano, via amostrador de Gibbs, utilizando-se o programa MTGSAM, desenvolvido por Van Tassel \& Van Vleck (1995), que assume que os efeitos sistemáticos seguem distribuição do tipo flat, que não considera qualquer conhecimento inicial a respeito desses efeitos, e que os componentes de (co)variância seguem distribuições de Wishart invertida. Assumiu-se que as distribuições dos componentes de (co)variância eram impróprias, atribuindo-se grau de crença igual a zero.

As amostras dos componentes de (co)variância foram obtidas de cadeias de 330.000 ciclos, em que os 30.000 ciclos iniciais foram descartados (burn-in) e as amostras foram retiradas a cada 100 ciclos (thinning interval), totalizando 3.000 amostras. Essa escolha baseou-se no fato de que, para aproximadamente $30 \%$ das análises, os resultados obtidos utilizando-se as cadeias com 330.000 ciclos (médias posteriores, regiões de alta densidade e amplitude) foram semelhantes aos obtidos com a utilização de cadeias maiores (1.100.000 ciclos, descarte dos primeiros 100.000 e amostragens a cada 1.000 ciclos).

A partir das amostras obtidas, foram calculadas as médias posteriores para os componentes de (co)variância e parâmetros genéticos, as regiões de alta densidade e o desvio-padrão associado a cada média posterior. As amostras dos componentes de (co)variância obtidas pelo Amostrador de Gibbs foram ordenadas de forma crescente, sendo calculados os intervalos entre as amostras 1 e 2.850 , 2 e $2.851, \ldots$, e 151 e 3.000 , de modo que cada intervalo continha $95 \%$ das amostras (2.850). O menor intervalo foi escolhido para apresentação e pode ser denominado intervalo de alta densidade, pois representa o menor intervalo contendo $95 \%$ das amostras obtidas.

Foram realizadas análises bicaráter de PD, P12 e PAD com as características TPR, ND10, NDT, QD10, QDTeQTPR. Os modelos estatísticos utilizados incluíram, além do efeito médio geral, os efeitos fixos de ano e mês de nascimento para PD, P12, TPR, ND10, NDT, QD10, QDT e QTPR, de ano, mês e idade da vaca $(4,5,6-8,>9)$ para $\mathrm{PAD}$, das covariáveis idade à desmama (efeito linear) e idade da vaca ao parto (efeitos linear e quadrático) para PD e da covariável idade aos 12 meses para P12. Os efeitos aleatórios considerados foram o efeito genético aditivo direto e o residual, para todas as características e o efeito de ambiente materno permanente (não correlacionado) para PD.

Foram consideradas as informações genealógicas de 8.440 animais, de forma que a matriz de parentesco continha 9.140 animais, incluindo, como base, os animais do grupo $5 / 8$ Charolês $+3 / 8$ Zebu, que, quando cruzados entre si, produziram os primeiros bimestiços denominados Canchim.

\section{Resultados e Discussão}

As médias gerais observadas foram iguais a $197 \mathrm{~kg}, 210 \mathrm{~kg}$, $500 \mathrm{~kg}, 3.050$ dias, 2,7 bezerros, $541 \mathrm{~kg}, 2,8$ bezerros, $549 \mathrm{~kg}$ e $54 \mathrm{~kg} /$ ano para PD, P12, PAD, TPR, ND10, QD10, NDT, QDT e QTPR, respectivamente. A média de TPR (3.050 dias; 8,3 anos; 100,3 meses) se assemelha àquela obtida por Barbosa et al. (2002) (8,25 anos), para o mesmo rebanho mas com arquivo contendo menos animais, e à observada por Mercadante et al. (2000) (104,8 meses) para um rebanho da raça Nelore. Tanida et al. (1988) obtiveram médias inferiores para as raças Hereford (7,40 anos) e Angus (6,68 anos). As médias observadas de ND10 (2,7 bezerros) e QD10 (541 kg) são ligeiramente superiores àquelas reportadas por Gianlorenço et al. (2003) (2,49 bezerros e 501,9kg), em razão da diferença nos arquivos. As médias de NDT ( 2,8 bezerros $)$, QDT (549 kg) e QTPR (54 kg/ano) foram superiores às observadas por Barbosa et al. (2002) (2,2 bezerros, $403,85 \mathrm{~kg}$ e $38,88 \mathrm{~kg} / \mathrm{ano}$ ) para o mesmo rebanho deste estudo, mas incluindo vacas que ainda não haviam sido descartadas. Tanida et al. (1988) obtiveram médias iguais a 3,46 e 3,66 bezerros desmamados durante toda a vida de vacas das 
raças Hereford e Angus, respectivamente. As médias registradas neste trabalho podem ser consideradas baixas, mas deve-se considerar o manejo reprodutivo e os critérios de descarte utilizados no rebanho durante sua existência e que a novilha que foi descartada sem desmamar nenhum bezerro foi considerada na análise com ND10, QD10, NDT, QDT e QTPR iguais a zero.

Na Tabela 1 encontram-se as médias posteriores e suas regiões de alta densidade para as herdabilidades e as correlações genéticas obtidas nas análises bicaráter de PD, P12 e PAD com TPR, ND10, QD10, NDT, QDT e QTPR.

As médias das estimativas de herdabilidade dos pesos das fêmeas foram iguais a 0,38 (PD), 0,40 (P12) e 0,54 (PAD), indicando que essas características possuem variação genética aditiva suficiente para boa resposta à seleção. Essas estimativas corroboram aquelas verificadas por Barbosa(1991), Alencar et al. (1993), Alencar et al. (1998), Silva et al. (2000) e Mello et al. (2002a), também para a raça Canchim.

As médias das estimativas de herdabilidade das características de longevidade e de produtividade, obtidas das análises bicaráter com PD, P12 e PAD, foram iguais a 0,22 (TPR), 0,22 (ND10), 0,24 (QD10), 0,23 (NDT), 0,23 (QDT) e 0,32 (QTPR), indicando também que é possível obter progresso genético pela seleção para essas características.

Para TPR, a média da herdabilidade neste trabalho $(0,22)$ confirma os resultados descritos por Gianlorenço et al. (2003), que, utilizando inferência bayesiana, em análise bicaráter com o peso aos 12 meses de idade de machos, estimaram média de herdabilidade de 0,24 . Analisando o mesmo conjunto de dados pelo método da máxima verossimilhança restrita, Gianlorenço et al. (2002) observaram média de herdabilidade para TPR de 0,11, enquanto Tanida et al. (1988), na raça Hereford, adotando o método dos quadrados mínimos, obtiveram o valor de 0,24 para a herdabilidade da idade do animal ao descarte e Mercadante et al. (2000) estimaram, na raça Nelore, os valores de 0,24 e 0,26 em análises bicaráter com os pesos à desmama e aos 12 meses de idade, respectivamente. Martinez et al. (2004a), na raça Hereford, determinaram herdabilidade de 0,15 para duração da vida produtiva (dias entre o primeiro parto e o descarte) permitindo ao animal viver até seis anos após o primeiro parto.

Para ND10 $(0,22)$ eQD10 $(0,24)$, as médias de herdabilidade neste trabalho foram abaixo dos valores encontrados por Gianlorenço et al. (2003) $(0,36$ e 0,41), utilizando inferência bayesiana, em análise bicaráter com o peso aos 12 meses de idade para machos. Para NDT, o valor encontrado $(0,23)$ foi similar ao $(0,22)$ obtido por Tanida et al. (1988), utilizando o método dos quadrados mínimos, em bovinos da raça Hereford. Martinez et al. (2004a), na raça Hereford, obtiveram herdabilidades de 0,21 e 0,18 para o número de bezerros desmamados e o peso à desmama acumulado em até seis anos após o primeiro parto, respectivamente, enquanto Martinez et al. (2004b) verificaram herdabilidades de 0,16 e 0,16 para o número de bezerros desmamados e o peso à desmama acumulado até os sete anos de idade, respectivamente.

As herdabilidades para QDT $(0,23)$ e QTPR $(0,31)$ foram inferiores àquelas de 0,29 e 0,36 , respectivamente, obtidas por Alencar et al. (2003).

A correlação genética $(-0,02)$ de PD e TPR indica que não há associação genética aditiva entre elas (Tabela 1). Mello et al. (2002b), analisando dados do mesmo rebanho, mas pelo método da máxima verossimilhança restrita,

Tabela 1 - Médias posteriores (região de alta densidade) das herdabilidades $\left(h^{2}\right)$ e das correlações genéticas $\left(r_{g}\right)$ obtidas nas análises bicaráter de PD, P12 e PAD (características 1) com TPR, ND10, QD10, NDT, QDT e QTPR (características 2)

Table 1 - A posteriori means (high density region) of heritabilities $\left(h^{2}\right)$ and genetic correlations $\left(r_{g}\right)$, obtained by the two-trait analyses of $P D, P 12$ and $P A D$ (Traits 1) with TPR, ND10, QD10, NDT, QDT and QTPR (Traits 2)

\begin{tabular}{|c|c|c|c|c|c|c|}
\hline \multirow[t]{3}{*}{$\begin{array}{l}\text { Caract. } 2^{1} \\
\text { Trait } 2^{1}\end{array}$} & \multicolumn{6}{|c|}{$\begin{array}{c}\text { Característica } 1^{1} \\
\text { Trait } 1^{1} \\
\end{array}$} \\
\hline & \multicolumn{2}{|c|}{$\mathrm{PD}$} & \multicolumn{2}{|c|}{ P 12} & \multicolumn{2}{|r|}{ PAD } \\
\hline & $\mathrm{h}^{2}{ }_{2}$ & $\rho_{\mathrm{g}}$ & $\mathrm{h}^{2}{ }_{2}$ & $\rho_{\mathrm{g}}$ & $\mathrm{h}^{2}{ }_{2}$ & $\rho_{\mathrm{g}}$ \\
\hline TPR & $0,21(0,10-0,32)$ & $-0,02(-0,31-0,25)$ & $0,22(0,12-0,33)$ & $0,26(0,00-0,53)$ & $0,22(0,12-0,33)$ & $-0,12(-0,40-0,20)$ \\
\hline ND 10 & $0,22(0,11-0,33)$ & $0,04(-026-0,35)$ & $0,22(0,12-0,32)$ & $0,10(-0,19-0,38)$ & $0,23(0,12-0,35)$ & $-0,29(-0,55-0,01)$ \\
\hline QD10 & $0,24(0,13-0,34)$ & $0,37(0,13-0,64)$ & $0,25(0,14-0,35)$ & $0,39(0,14-0,62)$ & $0,24(0,12-0,35)$ & $-0,13(-0,42-0,17)$ \\
\hline NDT & $0,23(0,12-0,34)$ & $-0,03(-0,32-0,22)$ & $0,23(0,13-0,34)$ & $0,14(-0,17-0,39)$ & $0,23(0,12-0,34)$ & $-0,25(-0,53-0,00)$ \\
\hline QDT & $0,22(0,13-0,33)$ & $0,20(-0,07-0,47)$ & $0,24(0,13-0,35)$ & $0,33(0,08-0,60)$ & $0,24(0,12-0,34)$ & $-0,16(-0,45-0,12)$ \\
\hline QTPR & $0,32(0,17-0,47)$ & $0,21(-0,04-0,47)$ & $0,31(0,18-0,45)$ & $0,28(0,02-0,53)$ & $0,32(0,19-0,44)$ & $-0,19(-0,44-0,09)$ \\
\hline
\end{tabular}

1 PD, P12, PAD, TPR, ND10, QD10,NDT, QDT e QTPR = pesos à desmama, aos 12 meses de idade e à idade adulta, tempo de permanência no rebanho, número e quilogramas de bezerros desmamados em até 10 anos de permanência no rebanho e durante todo o tempo de permanência e quilogramas de bezerros desmamados por ano de permanência no rebanho, em kg, kg, kg, dias, unidades, kg, unidades, kg e kg/ano, respectivamente. Número subscrito refere-se à característica.

$\mathrm{h}^{2}{ }_{1}$ (região de alta densidade) variando de 0,38 a 0,39 $(0,35-0,54)$ para PD, igual a 0,40 $(0,30-0,50)$ para P12 e variando de 0,53 a 0,55 (0,41-0,67) para PAD

${ }_{1}^{1} P D, P 12, P A D, T P R, N D 10, Q D 10, N D T, Q D T$ and $Q T P R=$ body weights at weaning, twelve months of age and adult age, herd life, number and kilograms of weaned calves up to 10 years of age and during herd life, and kilograms of calves weaned per year of herd life, in $\mathrm{kg}, \mathrm{kg}, \mathrm{kg}$, days, units, $\mathrm{kg}$, units, $\mathrm{kg}$ and $\mathrm{kg} / \mathrm{year}$, respectively. Number subscribed refers to trait.

$h^{2}{ }_{1}$ (high density region) from 0.38 to $0.39(0.35-0.54)$ for $P D$, equal to $0.40(0.30-0.50)$ for $P 12$, and from 0.53 to $0.55(0.41-0.67)$ for $P A D$. 
encontraram valor de baixa magnitude $(0,15)$, indicando que poucos genes de ação aditiva atuam nas duas características simultaneamente. Na raça Hereford, Tanida et al. (1988), utilizando o método dos quadrados mínimos e a correlação entre meio-irmãs paternas, estimaram correlação genética de 0,65 entre o tempo de permanência no rebanho e o peso à desmama das fêmeas.

Para P12 e TPR, a correlação genética foi igual a 0,26. Entretanto, a região de alta densidade sugere que este valor não é estatisticamente diferente de zero, o que indica que não há associação genética entre as características. Mello et al. (2002b), no mesmo rebanho, obtiveram os valores de 0,37, enquanto Mercadante et al. (2000) reportaram o valor de 0,61 para a correlação genética entre essas mesmas características em fêmeas da raça Nelore, ambos utilizando o método da máxima verossimilhança restrita. Gianlorenço et al. (2002), no mesmo rebanho estudado neste trabalho e pelo método de máxima verossimilhança restrita, reportaram correlação genética $(0,55)$ superior para $\mathrm{P} 12$ de machos e TPR de fêmeas, ao passo que Gianlorenço et al. (2003), utilizando inferência bayesiana, observaram o valor de 0,33 . Os resultados deste trabalho sugerem que a seleção para maior peso aos 12 meses de idade não deve resultar em mudanças no tempo de permanência das fêmeas no rebanho.

Para PAD e TPR, a correlação genética foi $-0,12$, com região de alta densidade de $-0,40$ a 0,20 , sugerindo a inexistência de associação genética entre as características.

As correlações genéticas de PD com ND10 $(0,04)$ e NDT $(-0,03)$ indicam que a seleção para PD não influencia o número de bezerros desmamados em até dez anos de idade e durante todo o tempo de permanência da vaca no rebanho. Com QDT $(0,20)$ e QTPR $(0,21)$, as correlações genéticas de $\mathrm{PD}$ apresentaram regiões de alta densidade, que sugerem que elas não são estatisticamente diferentes de zero. Com QD10 $(0,37)$, entretanto, a região de alta densidade $(0,13$ a $0,64)$ indica correlação genética estatisticamente diferente de zero e que a seleção para PD deve resultar em mais quilogramas de bezerros desmamados em até dez anos de idade. Tanida et al. (1988) observaram correlação genética favorável $(0,72)$ entre o número de bezerros desmamados e o peso à desmama de fêmeas da raça Hereford.

As correlações genéticas de P12 com ND10 $(0,10)$ e NDT $(0,14)$ apresentaram regiões de alta densidade que sugerem que elas não são estatisticamente diferentes de zero.Para QD10 $(0,39)$, QDT $(0,33)$ e QTPR $(0,28)$, entretanto, considerando-se as regiões de alta densidade, as correlações genéticas são estatisticamente diferentes de zero, indicando que a seleção para P12 deve afetar essas características. Gianlorenço et al. (2003), adotando inferência bayesiana em análise bicaráter com peso aos 12 meses de idade de machos, observaram valor de 0,38 (ND10) e 0,61 (QD10), demonstrando que a seleção para aumentar o peso deve resultar em maior número e quilogramas de bezerros produzidos pelas vacas em até dez anos de idade, sendo superiores aos reportados neste trabalho.

Das correlações genéticas de PAD com as características produtivas, apenas aquela com ND10 $(-0,29)$ pode ser considerada estatisticamente diferente de zero, em razão da região de alta densidade, de modo que o aumento do peso adulto das vacas pode reduzir o número de bezerros desmamados em até dez anos de idade.

$\mathrm{O}$ peso da vaca à desmama apresentou associação genética aditiva apenas com QD10 (Tabela 1), característica que envolve o peso de seus bezerros à desmama. Este fato parece normal, uma vez que a característica quilogramas é a soma dos pesos à desmama dos bezerros filhos das vacas em questão, ou seja, a soma de características de crescimento, como PD medido nas vacas. Para P12, no entanto, essa associação aconteceu com todas as características que envolvem quilograma de bezerros. Para o peso adulto, a correlação genética foi negativa com ND10, o que pode ser indicativo de que as vacas com potencial genético para maior peso adulto são desfavorecidas nessa característica.

Em vista desses resultados, a seleção para maiores pesos à desmama e aos 12 meses de idade com o propósito de aumentar a produtividade do rebanho deve ser feita com a preocupação de não aumentar o peso à idade adulta das vacas, o que é possível, pois a relação genética entre esses dois grupos de pesos, apesar de desfavorável, não é perfeita.

\section{Conclusões}

Os pesos à desmama, ao ano e à idade adulta e as características de longevidade e produtivas estudadas possuem variação genética aditiva suficiente para justificar sua inclusão nos programas de melhoramento de bovinos da raça Canchim.

A seleção para maiores pesos à desmama e ao ano na raça Canchim deve resultar em mais quilogramas de bezerros desmamados pelas vacas, sem reduzir seu tempo de permanência no rebanho e o número de bezerros produzidos. Entretanto, o aumento do peso adulto da vaca pode refletir em menor número de bezerros desmamados até dez anos de idade.

\section{Literatura Citada}

ALENCAR, M.M. Critérios de seleção em bovinos de corte no Brasil. In: SIMPÓSIO NACIONAL DE MELHORAMENTO ANIMAL, 4., 2002, Campo Grande. Anais... Campo Grande: Sociedade Brasileira de Melhoramento Animal, 2002. (CD-ROM). 
ALENCAR, M.M.; BARBOSA, P.F.; BARBOSA, R.T. Parâmetros genéticos para peso e circunferência escrotal em touros da raça Canchim. Revista Brasileira de Zootecnia, v.22, n.4, p.572583, 1993

ALENCAR, M.M.; TORAL, F.L.B.; MELLO, S.P. et al. Parâmetros genéticos para peso aos doze meses de idade, idade ao primeiro parto e características de produtividade em fêmeas da raça Canchim. In: CONGRESSO NACIONAL DE GENÉTICA, 47. 2003, Águas de Lindóia. Anais... Águas de Lindóia: Sociedade Brasileira de Genética, 2003. (CD-ROM).

ALENCAR, M.M.; TREMATORE, R.L.; BARBOSA, P.F. et al. Efeitos da linhagem citoplasmática sobre características de crescimento em bovinos da raça Canchim. Revista Brasileira de Zootecnia, v.27, n.2, p.272-276, 1998.

ANUALPEC 2003: Anuário da pecuária brasileira. São Paulo: FNP Consultoria \& Comércio: Editora Argos, 2003. 400p

BARBOSA, P.F. Análise genético-quantitativa de características de crescimento e reprodução em fêmeas da raça Canchim. Ribeirão Preto: Universidade de São Paulo, 1991. 237p. Tese (Doutorado em Genética) - Universidade de São Paulo, 1991.

BARBOSA, P.F.; ALENCAR, M.M.; SILVA, A.M. Peso à maturidade, taxa de maturação e eficiência produtiva em fêmeas de bovinos da raça Canchim. Arquivo Brasileiro de Medicina Veterinária e Zootecnia, v. 54, n.5, p.510-517, 2002.

GIANLORENÇO, V.K.; ALENCAR, M.M.; MELLO, S.P. et al. Correlações genéticas entre peso e perímetro escrotal de machos com o tempo de permanência de fêmeas em um rebanho da raça Canchim In: SIMPÓSIO NACIONAL DE MELHORAMENTO ANIMAL, 4., 2002, Campo Grande. Anais... Campo Grande: Sociedade Brasileira de Melhoramento Animal, 2002, p-96. (CD-ROM)

GIANLORENÇO, V.K.; ALENCAR, M.M.; TORAL, F.L.B. et al. Herdabilidades e correlações genéticas de características de machos e fêmeas, em um rebanho bovino da raça Canchim. Revista Brasileira de Zootecnia, v.32, n.6, p.1587-1593, 2003 (supl. 1).

LANNA, D.P.; PACKER, I.U. Eficiência biológica e econômica de bovinos de corte In: WORKSHOP QUALIDADE DA CARNE E MELHORAMENTO GENÉTICO DE BOVINOS DE CORTE, 1., 1998, São Carlos. Anais... São Carlos: Embrapa-CPPSE/São Paulo: FUNDEPEC/Campo Grande: Embrapa-CNPGC, 1998. p.172.

MARTINEZ, G.E.; KOCH, R.M.; CUNDIFF; L.V. et al. Genetic parameters for six measures of lenght of productive life and three measures of lifetime production by $6 \mathrm{yr}$ after first calving for Hereford cows. Journal of Animal Science, v.82, p.1912$1918,2004 a$.
MARTINEZ, G.E.; KOCH, R.M.; CUNDIFF; L.V. et al. Number of calves born, number of calves weaned, and cumulative weaning weight as measures of lifetime production for Hereford cows. Journal of Animal Science, v.82, p.1903-1911, 2004b.

MELLO, S.P.; ALENCAR, M.M.; SILVA, L.O.C. et al. Estimativas de (co)variâncias e tendências genéticas para pesos em um rebanho Canchim. Revista Brasileira de Zootecnia, v.31, n.4, p.17071714, 2002a

MELLO, S.P.; GIANLORENÇO, V.K.; ALENCAR, M.M. et al. Correlações genéticas entre pesos e tempo de permanência de fêmeas em um rebanho da raça Canchim In: SIMPÓSIO NACIONAL DE MELHORAMENTO ANIMAL, 4., 2002, Campo Grande, MS. Anais... Campo Grande: Sociedade Brasileira de Melhoramento Animal, 2002b, p.96-98. (CD-ROM).

MERCADANTE, M.E.Z.; LÔBO, R.B.; OLIVEIRA, H.N. Estimativas de (co)variâncias entre características de reprodução e de crescimento em fêmeas de um rebanho Nelore. Revista Brasileira de Zootecnia, v.29, n.4, p.997-1004, 2000.

SILVA, A.M.; ALENCAR, M.M.; FREITAS, A.R. et al. Herdabilidade e correlações genéticas para peso e perímetro escrotal de machos e características reprodutivas e de crescimento de fêmeas, na raça Canchim. Revista Brasileira de Zootecnia, v.29, n.6, p.2223-2230, 2000 (supl. 2).

TALHARI, F.M.; ALENCAR, M.M.; MASCIOLI, A.S. et al. Correlações genéticas entre características produtivas de fêmeas em um rebanho da raça Canchim. Revista Brasileira de Zootecnia, v.32, n.4, p.880-886, 2003.

TANIDA, H.; HOHENBOKEN, W.D.; DENISE, S.K. Genetic aspects of longevity in Angus and Hereford cows. Journal of Animal Science, v.66, n.3, p.640-647, 1988.

Van TASSEL, C.P.; Van VLECK, L.D. A manual for use of MTGSAM. A set of fortran programs to apply gibbs sampling to animal models for variance component estimation. Lincoln: Department of Agriculture/Agriculture Research Service, 1995. 86p. 